\title{
ESPAÑA
}




\section{Inmigración interior e inmigración exterior}

\section{Carlota Solé}

Universitat Autònoma de Barcelona. Departament de Sociologia

08193 Bellaterra (Barcelona). Spain

Carlota.Sole@uab.es

\section{Resumen}

El artículo compara sistemáticamente la situación de la inmigración de españoles y extracomunitarios a Cataluña atendiendo a los flujos, a la competencia en el mercado laboral, al proceso de integración y al papel que tiene el conocimiento de la lengua en el proceso sociocultural de integración en una sociedad sentida y definida como nación por una mayoría de sus habitantes. En último lugar, se añade también en el artículo un apartado sobre inmigración femenina para dar mayor coherencia al número monográfico de la revista.

Palabras clave: inmigración interior, inmigración exterior, integración, lenguas, inmigración femenina.

\section{Abstract. Internal and External immigration}

The article systematically compares the situation of Spanish and non-EU migrants in Catalonia (Spain). It analyses the composition of flows, the competence in the labour market, the process of integration and the role of language in the sociocultural process of integration of immigrants. The question of immigrant women's situation is added in order to give a better cohesion to the monograph.

Key words: internal immigration, external immigration, integration, language, women's immigration.

\section{Sumario}

1. Cataluña, sociedad de emigración e inmigración

2. Competencia en el mercado laboral

3. Integración en Cataluña
4. Lengua e integración

5. La inmigración femenina

Bibliografía 


\section{Cataluña, sociedad de emigración e inmigración}

Cataluña ha sido históricamente un centro de atracción y confluencia de gente de diversa procedencia geográfica. Muchas veces ha interrumpido el viaje de tantos emigrantes en una tierra de paso hacia Europa. Los flujos de población y los intercambios culturales la han enriquecido como sociedad y han reforzado a lo largo de los siglos el doble sentimiento y el papel, hospitalario y al mismo tiempo receloso, de no perder el sentido de identidad social, cultural y lingüística.

Tradicionalmente la inmigración masiva a Cataluña ha procedido de otras zonas de España. La inmigración interior se ha producido en olas periódicas paralelas a la construcción de obras de infraestructura urbana o de expansión económica general. Éste es el caso de la década de 1960, y más concretamente los años 1962 y 1963, en los que la llegada de andaluces, extremeños o gallegos fue explosiva desde el punto de vista demográfico y dio lugar más tarde a importantes problemas de especulación del suelo, vivienda, discriminación laboral en razón de una menor formación profesional, integración sociocultural, etc., a resolver.

Pero recientemente, desde los años ochenta, la inmigración interior se complementa con la ligeramente creciente inmigración exterior. A pesar de que la expresión «inmigrantes» sea aún atribuida, semánticamente y de forma exclusiva, a las personas procedentes de otras zonas de España e instaladas en Cataluña, no se puede obviar la realidad de la inmigración procedente de países llamados "del Tercer Mundo». Estos inmigrantes extranjeros extracomunitarios se han instalado desde su llegada a la ciudad de Barcelona y las comarcas más cercanas a la capital catalana, y en menor proporción en las comarcas de Lérida, Tarragona y Gerona. La inmigración de africanos (Gambia, Senegal, Camerún, Guinea Bissau, Nigeria), marroquíes, libaneses, pakistaníes, filipinos, entre los grupos más numerosos, se ha producido de una forma más notoria durante los últimos diez años, existiendo en proporciones exiguas en décadas anteriores.

España ha dejado de ser un país de emigración para empezar a ser un país de inmigración, pero Cataluña ha sido siempre una sociedad receptora de inmigrantes. En general esta tradición histórica ha hecho que, siendo tierra de paso, no se produjera un rechazo manifiesto a los trabajadores del llamado Tercer Mundo cuando éstos llegaron a principios de la década de los ochenta. A medida que avanzaba la década y se incrementaba el número de africanos occidentales, marroquíes o asiáticos, aumentaron las tensiones que, en los años noventa, se han manifestado en conflictos raciales, muestras de actitudes xenófobas, en diversas comarcas, municipios y rincones de Cataluña. Paralelamente, se produce un afianzamiento del asociacionismo entre estos colectivos, una mayor concienciación por parte de la población autóctona de la presencia de estas personas y un intento por parte de las instituciones públicas y privadas de solucionar los problemas de estos colectivos.

Cataluña, especialmente la ciudad de Barcelona, se ha convertido en un polo de atracción de migraciones internacionales (actualmente la segunda 
comunidad autónoma en número de permisos de trabajo a extranjeros no comunitarios), a la vez que ha presenciado la consolidación de las migraciones internas acaecidas en décadas anteriores. Justamente el retroceso de las migraciones interiores, la sedentarización de las segundas generaciones y el retorno en algunos casos de las primeras migraciones interiores perfilan el preámbulo al inicio de la llegada de trabajadores procedentes de países del llamado Tercer Mundo.

De acuerdo con el análisis cuantitativo del perfil de la inmigración española (a partir de las estadísticas totales de extranjeros) A. Izquierdo (1992) distingue tres fases: 1) aumento regular importante (años 60-70); 2) un rápido crecimiento (80-85) y 3) una fase de aceleración (85-89). En ésta última tiene incidencia la Ley orgánica de 1985 y la regularización de extranjeros en un contexto de crecimiento económico y de apertura internacional. La Ley de extranjería (7/85) codifica la entrada, la residencia, el trabajo y los derechos de los extranjeros en España. Teniendo presente el perfil español, la mayoría de extranjeros llegaron a España entre 1990 y 1991 (un 51,3\%) (Aragón y Chozas, 1993, p. 104).

Para examinar la situación de los inmigrantes en Cataluña, y en concreto de Barcelona, es necesario recurrir a la Constitución española (que atribuye al Estado español la competencia exclusiva en materia de nacionalidad, inmigración, emigración, derecho de asilo y relaciones internacionales), así como al traspaso de competencias al Gobierno autonómico (determinadas competencias legislativas y de ejecución en materias que afectan a la inmigración, que - junto con los municipios- se encuentra en el ámbito de la atención social). Debemos recurrir también a la concesión de permisos de residencia y de trabajo dependientes del Ministerio de Interior y de sus órganos provinciales. En materia de visados es competente el Ministerio de Asuntos Exteriores y en materia de expulsiones, el de Justicia.

A partir de 1980, Cataluña experimenta, por primera vez, un saldo migratorio negativo, debido al impacto de la crisis económica y al retorno de los inmigrantes interiores a sus zonas de origen en el contexto del reequilibrio de las regiones españolas. Desde ese momento podemos hablar del asentamiento de un nuevo modelo común a otras zonas del litoral mediterráneo y a la Comunidad Autónoma de Madrid, caracterizado por la inmigración progresiva de trabajadores extracomunitarios y de países de Europa del Este, especialmente de países con los que España tenía vínculos coloniales, como es el caso de los países latinoamericanos, Marruecos y Filipinas. La diferencia clave entre esta nueva inmigración y la interior (1950-1975), en términos económicos es que la inmigración actual no es una inmigración masiva y que no está determinada por la incorporación de trabajadores a una economía expansiva con necesidad de mano de obra en muchos sectores económicos, sino en aquéllos más regresivos o con mayores dificultades de cambio tecnológico. En términos legales, la inmigración interior la componen ciudadanos de un mismo Estado. En cambio, los inmigrantes extracomunitarios no tienen ninguna cobertura legal a priori para residir y trabajar en España y, en su contexto, en 
Cataluña. Estos dos aspectos clave: inserción de los inmigrantes en una economía expansiva o recesiva y ciudadanía o no ciudadanía española, condicionan la integración sociocultural de los inmigrantes interiores y extracomunitarios en la sociedad receptora.

\section{Competencia en el mercado laboral}

Dado un mercado de trabajo dual (Sabel, 1985): por un lado, casi totalmente transparente para los trabajadores autóctonos, arraigados en el país, especializados, con acceso a la información, know how; y, por otro lado, casi totalmente opaco para otro grupo de trabajadores inmigrantes, no especializados, con casi nula información sobre las condiciones de trabajo, salarios, acción sindical en la sociedad receptora; existe competencia entre los trabajadores autóctonos e inmigrantes que en los años 1960 llegaron a Cataluña desde el sur de España (inmigrantes interiores) y los trabajadores procedentes de los países del denominado Tercer Mundo, llegados a Cataluña a finales de los años setenta y la década de los ochenta (inmigrantes extranjeros). Los trabajadores inmigrantes extranjeros tienen un papel de ejército de reserva respecto al resto de trabajadores españoles. La diferencia más notable entre ambos colectivos, actores sociales o jugadores (en terminología de la teoría de los juegos), es que los primeros deben legalizar su situación como residentes y trabajadores en España, mientras que los segundos son ciudadanos del Estado español.

La progresiva especialización de las actividades productivas da lugar a una segmentación muy fuerte del mercado de trabajo que puede llegar a correlacionarse estrechamente con los grupos étnico-culturales. Así, las tareas más sucias, rutinarias, repetitivas, etc., que corresponden a ocupaciones que se sitúan en los estratos más bajos de la estructura ocupacional son rechazadas por los trabajadores autóctonos y, también progresivamente, en la medida en que experimentan movilidad ocupacional, por los trabajadores inmigrantes españoles. Otras actividades que exceden a la mano de obra disponible son también realizadas por los extranjeros inmigrantes de los países del Tercer Mundo. Ahora bien, existe una franja de actividades y tareas realizadas por trabajadores semiespecializados o poco cualificados, generalmente inmigrantes interiores, que temen la competencia de los inmigrantes procedentes del Tercer Mundo. Cabe reconocer que existen tres situaciones en el mercado de trabajo que pueden conducir a la discriminación: substituibilidad de la mano de obra, complementariedad y competencia.

De acuerdo con nuestras investigaciones sobre la inmigración en Cataluña desde el resto de España $(1981,1988)$, en conjunto, la población autóctona goza de un nivel medio de estudios superior al de los inmigrantes, posee mejor cualificación profesional y goza de mejor situación laboral dentro de la estructura ocupacional. La valoración que hacen los inmigrantes interiores de trabajar en Cataluña en comparación con otras partes de España, aun siendo positiva, recoge esta diferencia, por cuanto las posibilidades de promoción aparecen más lejanas para ellos que para los catalanes de origen. 
En las empresas y centros de trabajo en general, hay una tendencia a concentrarse trabajadores, cuadros medios, jefes, gerentes, castellano y catalanoparlantes, por separado. De ahí que casi tres cuartas partes de la población activa inmigrante afirme que no precisa saber catalán en su empresa y que sea la cualificación en el trabajo el criterio más reconocido como válido para encontrar y cambiar de empleo en Cataluña, más que el de conocer la lengua, ser joven y otros.

Pese a ser un proceso lento, amoldarse al trabajo industrial es el objetivo principal que se proponen los inmigrantes interiores de primera generación en Cataluña, procedentes de un medio agrícola y rural. La penetración de la población activa inmigrada en la estructura ocupacional es muy notoria, en lo referente al tipo de ocupación, facilidad de encontrar y cambiar de trabajo en los últimos años, etc., en comparación a la población activa autóctona.

Las posibilidades de promoción en el puesto de trabajo y de asumir un nivel de estatus social superior se han relacionado en los últimos tiempos, por parte de la opinión pública, con el hecho de conocer la lengua catalana y utilizarla en el puesto de trabajo. Este elemento debe considerarse bajo la condición de la real concentración de catalanoparlantes y castellanoparlantes en empresas y centrales laborales de las diferentes ramas de la producción. No sólo en relación con la ocupación en la tierra de origen, sino también con el primer trabajo realizado, es interesante ver los cambios que afectan a la población inmigrada en edad de trabajar, en comparación con la población nacida en Cataluña, incluidos los hijos de los inmigrantes.

Trabajar en Cataluña, a diferencia de otros lugares de España, es considerado como más seguro, por la estabilidad en el trabajo y la regularidad en los salarios. La característica más clara es, sin embargo, la de que en Cataluña hay posibilidades de promoción. Ésta es la opinión casi mayoritaria de las personas nacidas en Cataluña; especialmente las de clase alta y media-alta. Los obreros, especialmente los inmigrantes, no sienten en la misma medida las facilidades para promocionarse, como los autóctonos, aún afirmando el carácter de seguridad en el lugar de trabajo y salarios más regulares que han encontrado aquí. Además de estas notables diferencias, aproximadamente un tercio de la población entrevistada, inmigrada o no, de cualquiera de las clases sociales con las cuales se identifican subjetivamente los entrevistados, creen que no hay diferencia entre trabajar en Cataluña o en otras partes del territorio español. La cuestión de tener que conocer la lengua es considerada como importante sólo por un $10 \%$, aproximadamente, de la población total del Área Metropolitana de Barcelona donde se realizó la encuesta, como también, de la población activa de la zona (Solé, 1988).

Comparando la situación ocupacional actual y la que tuvieron los inmigrantes interiores al llegar a Cataluña y en el lugar de origen, podemos analizar las posibilidades de movilidad social a través del trabajo que han conseguido al abandonar sus tierras. Se observa que el traspaso de peones y propietarios agrícolas hacia actividades industriales o de servicios, afecta a los inmigrantes de forma considerable. Una vez en Cataluña se ocupan en el primer trabajo 
como obreros sin cualificar y, en segundo lugar, como obreros cualificados, para más tarde convertirse en trabajadores independientes o autónomos. La ocupación como administrativos y ocupaciones de tipo medio es, en tercer lugar, la que absorbe a más población inmigrada, una vez llegados a Cataluña y en el momento de la encuesta (1983).

La ocupación se relaciona todavía estrechamente con el nivel de estudios. La cualificación en el trabajo proviene del nivel y tipo de conocimientos y formación profesional recibidas. Así, el número de personas sin estudios es reducido, mientras que las personas con formación primaria son muchas, especialmente entre los obreros. La clase media inmigrada contiene un número igualmente elevado de personas con escuela de primer nivel. El bachillerato o estudios de secretariado atrae más a los autóctonos que a los inmigrantes. Las diferencias se hacen más notorias entre los miembros de la clase media, a favor de los catalanes de origen y de los inmigrantes en lo que respecta a la clase obrera. La consistencia por clase social en relación con el nivel de estudios asumido es muy marcada, de manera que cuanto más elevada es la clase social, mayor es la proporción de personas con nivel universitario y al revés, cuanto más baja la clase social, más elevado el número de nacidos y no nacidos en Cataluña que tienen estudios primarios. En conjunto los autóctonos tienen un nivel de estudios más alto que los inmigrantes (Solé, 1981, 1988). Entre la población extracomunitaria inmigrada, en cambio, el nivel de educación es, en muchos casos, superior al requerido para llevar a cabo una actividad laboral. Inmigran a nuestro país personas con un nivel medio de formación y se emplean en el servicio doméstico, la construcción o la agricultura como peones. Esta inconsistencia es realmente evidente en el caso de las mujeres inmigrantes (Solé, 1994).

\section{Integración en Cataluña}

Las dificultades para obtener un puesto de trabajo (y el condicionante previo de un permiso para residir y para trabajar), la discriminación en el acceso al mundo laboral y la potencial dualidad del mercado de trabajo, ponen sobre el tapete la cuestión de la real integración de los inmigrantes en la sociedad receptora.

Existen grados de integración social y cultural que están condicionados por la raza o el color de la piel. No por irracional menos real, ese elemento diferencial entre los colectivos de trabajadores extranjeros afecta su proceso de integración sociocultural. En líneas generales se cumplen las premisas de ese proceso al igual que en el caso de los inmigrantes procedentes de otras regiones españolas a Cataluña. Pero existen diferencias importantes que lo dificultan en el caso de los inmigrantes extranjeros. En primer lugar, no son ciudadanos de un mismo Estado. Por el contrario, se enfrentan cotidianamente a las restricciones de la Ley de extranjería promulgada en 1985 que regula legalmente la entrada y permanencia de los extranjeros en España y el Reglamento de 1996 y, a partir de abril de 2000, la nueva Ley de extranjería, aprobada en diciembre de 
1999. Por otro lado, los contrastes culturales y lingüísticos al emigrar a Cataluña son mayores evidentemente para los emigrantes de origen africano o asiático que de origen español. En tercer lugar, las diferencias por raza son inexistentes en el caso de la inmigración interior. Estos tres factores — ciudadanía, cultura, raza - dificultan la pronta integración de este tipo de inmigración, pero no la imposibilitan, bajo el supuesto de que la población autóctona revise necesariamente sus ideas preconcebidas y su actitud en relación con el beneficio que supone la inmigración para la sociedad receptora.

El concepto de integración implica que los inmigrantes se aproximen, sea cual sea su procedencia geográfica y teniendo en cuenta una situación objetiva de clase y una autoidentificación subjetiva con la sociedad receptora, a la situación y posición social de los autóctonos. Ahora bien, en virtud del mismo razonamiento, puede significar que los inmigrantes reclamen la consideración o el reconocimiento de sus propias características étnicas y culturales, y al mismo tiempo acepten la forma de vida, las instituciones y los símbolos de la sociedad receptora. Si este reconocimiento se materializa puede llevar a conflicto con las instituciones ya enraizadas en la sociedad o bien, al contrario, puede derivar en consenso sobre las diferencias existentes (Solé, 1995).

La comunidad receptora puede, sin embargo, desarrollar mecanismos selectivos en la acogida de las personas, extracomunitarias o no, que se instalen en su seno. Este hecho agrava los potenciales conflictos intra e interclase que se ven reforzados por la dimensión étnica como criterio discriminador, al comportar diferentes niveles de oportunidades previas a la educación y a la práctica profesional en comparación con la población autóctona. Como en otras sociedades receptoras de inmigración, en Cataluña hay diferenciaciones por segmentos o categorías de la estructura ocupacional informalmente vinculados y cubiertos por grupos étnicos diferentes que, siendo minoritarios en el conjunto de la población de Cataluña, se han de entrecruzar con la variable llamada «clase social».

Este hecho refuerza la necesidad de los inmigrantes de decantarse por las instituciones ya existentes en los ámbitos económicos, cultural, social y otros. También hay una razón pragmática que explica la esporádica existencia, por el momento, de conflicto abierto en la convivencia de las dos subpoblaciones, autóctona y no autóctona, en la sociedad catalana. Atendiendo a la estructura ocupacional existente en la actualidad, a ningún inmigrado en concreto le interesa enfrentarse a otros inmigrantes o a los autóctonos, por cuestiones lingüísticas o culturales. Conocer mínimamente la lengua catalana y aceptar las costumbres, símbolos e instituciones no hará más que aumentar sus posibilidades individuales de competir en el mercado de trabajo y de participar en la vida social y cultural de la ciudad.

De la misma forma, a ningún inmigrado, extranjero o no, le interesará organizarse en una acción coordinada con otras personas inmigradas con el fin de obtener una hipotética ventaja social o cultural en el reconocimiento de su propia entidad étnica o cultural. El cálculo racional de sus propias posibilidades de integración sociocultural los llevará a aceptar los condicionamientos 
culturales y lingüísticos dominantes en este momento en la comunidad catalana. El conflicto devendrá generacional, en el sentido que los inmigrantes de primera generación viven en dos sistemas referenciales, el de la sociedad de origen y el de la sociedad receptora; mientras que los de segunda generación viven entre dos sistemas referenciales, no tanto estructuralmente hablando como generacionalmente. El proceso societal y cultural de integración de la inmigración en Cataluña en la actualidad no conlleva situaciones graves de discriminación en función de la lengua o la raza, pero sí persistentes en relación con otras variables como el salario, las condiciones laborales o las relaciones en el lugar de trabajo y en la sociedad.

\section{Lengua e integración}

La integración sociocultural en la sociedad receptora exige inevitablemente la adquisición de un conocimiento mínimo de la lengua propia y/o oficial en el territorio como vía de comunicación y de intercambio. En el caso de Cataluña, como sociedad receptora, la coexistencia de dos lenguas exige un mayor esfuerzo a los inmigrantes interiores y exteriores. La diferencia en la aceptación de esta situación lingüística para unos y otros está en la época distinta en que los inmigrantes interiores y los exteriores se instalaron en Cataluña. Así, en el primer caso, la inmigración de andaluces, extremeños, gallegos y otros españoles tiene lugar en plena época franquista (años sesenta), época en que aprender el catalán o en catalán en la escuela era imposible, por prohibido, y hablarlo en privado era tolerado.

En aquella época, aunque la existencia de una lengua y cultura propias fuera vagamente conocida por el inmigrante, por la misma opresión política y social que había sufrido el país, su contacto con la lengua y cultura catalanas era sistemáticamente obstruido, a partir del mismo momento de acomodarse a la vida industrial y urbana en Cataluña. El contacto con la realidad catalana era prácticamente inexistente en la vida diaria o a través de los medios de comunicación, además de la carencia de escuelas y escasa enseñanza en ellas del y en catalán.

La integración en una sociedad con unas características culturales propias se veía dificultada por obstáculos que no eran los hipotéticos y potenciales prejuicios del inmigrado o su actitud defensiva ante el hecho catalán, sino por hechos objetivos y deficiencias estructurales de la sociedad catalana. Estos hechos objetivos son el gran volumen de la inmigración y la necesidad de los inmigrantes de concentrarse tanto en el lugar de trabajo como en el de residencia. El aislamiento en los barrios, el escaso contacto con catalanes en los lugares públicos, o contactos con la Administración, que se realizaban casi automáticamente en la lengua oficial (hasta 1978, sólo la castellana), impedía también la concienciación de muchos inmigrantes sobre la utilidad de aprender el catalán, al no sentirse acuciados directamente por la necesidad de emplearlo como lengua de relación o de comunicación. Las deficiencias estructurales eran, por un lado, la falta de escuelas y la imposibilidad de educarse y formarse profe- 
sional y culturalmente; por otro, la impermeabilidad de los modernos medios de comunicación, controlados por el Estado —en especial, en la época franquista - a la lengua y a la cultura catalanas.

Esta situación de precariedad estructural tuvo como efecto, el desarraigo de la propia cultura que afecta a la población autóctona con respecto a su lengua y cultura, y la no identificación con ellas de la población inmigrante. En el primer caso, es conocida la realidad diglósica que escondía el bilingüismo ambiental predominante en Cataluña, en los años cincuenta y sesenta por la amplia opresión de la lengua y cultura catalanas durante siglos, y su feroz represión en las dos décadas anteriores. En el segundo caso, la población inmigrante se encontraba ante el problema de desconocimiento y no-identificación con la cultura catalana que podía, a veces, manifestarse en la reproducción parcial y/o esporádica de formas de vida de la comunidad de origen, en lugar de adoptar, de manera natural y sin imposiciones, pautas culturales de la sociedad receptora.

Por otra parte, sectores importantes del campesinado y pequeña burguesía urbana de Cataluña son de hecho monolingües catalanas, llegando a entender el castellano sin apenas hablarlo o escribirlo. A su vez, para los inmigrantes ya afincados o recientemente llegados a Cataluña, la realidad diglósica que tan profundamente afecta a los catalanoparlantes, ha podido servir indirectamente para paliar el contraste y conflicto entre dos lenguas en contacto, al tiempo que no ha motivado al inmigrante a conocer una segunda lengua. El inmigrante interior no siente la necesidad de aprender el catalán, ni tampoco el apremio de reafirmarse en su propia lengua, al ser el castellano la lengua dominante y oficial. Esta afirmación debería matizarse en el caso de inmigrantes gallegos, vascos, y, en general, de personas cuya lengua materna no fuera el castellano.

En aquella época la lengua del entorno no era el catalán, coexistiendo con el castellano, y resultaba difícil neutralizar la existencia de una lengua dominante: el castellano. El catalán no podía ser adoptado por los inmigrantes de forma natural. La lengua como vehículo esencial de transmisión de cultura, en su contenido y forma, no se transformaría fácilmente en un medio de integración sociocultural. En Cataluña, la exigencia de la restauración oficial completa de la lengua entrañaba la cooficialidad obligada y asumir el aprendizaje del catalán en la escuela.

Partiendo de la vinculación entre cultura y lengua, no puede afirmarse que exista correlación entre clase social y habla catalana o castellana, puesto que una clase social no se define por la lengua. En realidad, en Cataluña el catalán no es, ni ha sido únicamente, la lengua de la burguesía; es y ha sido también la lengua de las clases populares autóctonas, incluida gran parte del proletariado de hoy. Por otra parte, no puede afirmarse que el hecho de no haber aprendido el catalán signifique una menor integración a otros niveles, exceptuando el idiomático.

Aunque es muy probable que en la segunda y en sucesivas generaciones, se produzca un contacto más frecuente y directo de los inmigrantes con la len- 
gua catalana o, por lo menos, que existan mayores posibilidades de que se produzca este contacto, el conocimiento del idioma y su uso son evidentemente necesarios, en la tarea de integración en un país de inmigración masiva como es Cataluña y, en especial, si la población inmigrante ha abandonado en su mayoría la idea de retorno a la región de origen. En el caso del inmigrante, la escuela le proporciona los instrumentos necesarios para acomodarse al trabajo industrial y a las condiciones de vida de la sociedad industrial urbana catalana, aun para aquellos inmigrados e hijos de inmigrantes que puedan sentirse catalanes por haber nacido, vivir y/o trabajar en Cataluña pero no hablan el catalán y desconozcan las raíces históricas y políticas de la reivindicación lingüístico-nacional catalana.

Dos décadas más tarde, la situación de coexistencia del catalán y el castellano en esta zona de España ha cambiado. A partir de la aplicación de la Ley de normalización lingüística de 1983 se garantiza la enseñanza del catalán, reforzada por la Ley de política lingüística del 7 de enero de 1998, en paridad o complementariedad con la del castellano, en todas las escuelas de Cataluña. Todos los alumnos, incluidos los hijos de los inmigrantes extracomunitarios que fluyen hacia nuestro país desde principios de los años ochenta, reciben educación en o de catalán. De acuerdo con un estudio (Solé, 1999) llevado a acabo en el barrio barcelonés del Raval, donde se concentra gran número de inmigrantes, pese al desconocimiento inicial de la existencia del catalán al llegar aquí, la necesidad de conocer esta lengua se hace necesaria para los inmigrantes del Tercer Mundo, no sólo por razones laborales (es decir, tener más probabilidades de encontrar y cambiar de trabajo) sino también de convivencia («a los catalanes les gusta que les hablen en catalán») y acomodación a las nuevas condiciones de vida y de trabajo del entorno («si hablo catalán, muy contentos: me compran más», entrevista número 12).

Según este estudio, ninguno de los entrevistados tenía información sobre el grado de extensión del catalán en una zona de la geografía española previamente a inmigrar a Barcelona. La mayor parte pensaban que se hablaba el castellano como en toda España y algunos creían que en Cataluña se hablaba el inglés. Esta falta de información se correlaciona positivamente con el nivel de estudios y el número de países a los cuales emigraron anteriormente las personas entrevistadas. Cuanto más elevados son ambos indicadores, más información se tiene sobre el catalán. El hecho de proceder de países donde coexisten diversas lenguas y culturas, y de haber conocido muchos otros países antes de instalarse en Barcelona, ayuda también a aceptar el aprendizaje necesario, aún siendo pasivo y no activo, de la lengua del país donde se vive, siempre con la finalidad de obtener un beneficio inmediato de una comunicación más fluida con el entorno catalanoparlante: «estoy aquí en Barcelona y sirve saber catalán. Puedo vender más cosas» (entrevista número 15).

Pese a que el aprendizaje en la escuela es prácticamente imposible para estas personas laboralmente activas, cumpliendo horarios muy extensos y sometidos a condiciones de trabajo precarias, el contacto en la calle y a través de las cadenas de televisión catalanas, facilita el conocimiento de la lengua cata- 
lana y su uso. Prueba de la extensa audiencia de los programas de TV3 y Canal 33 son las exhaustivas respuestas afirmativas de las cuarenta entrevistas, que conectan estos canales para ver películas y programas informativos de su interés, con la excepción de algunas personas que declaran escuchar los noticiarios en castellano. La televisión más que la radio es, pues, igual que para la inmigración interior, una vía pasiva, pero eficaz, de conocimiento de la lengua, al actuar en el tiempo libre y no implicar ninguna imposición por razones de trabajo.

El incentivo de utilizar el catalán se encuentra vinculado a la actividad económica. Las personas que tienen un trabajo manual (mecánico de coches, lampista, montador de bicicletas, soldador) o que no implica el contacto con el público (cocinero, lavaplatos en un restaurante, repartidor en camión) o que se realice en un entorno laboral castellanoparlante (puesto de trabajo donde se concentran trabajadores de inmigración interior: andaluces, gallegos, etc.) no sienten objetivamente la necesidad de su uso. Así, la gran mayoría de los entrevistados manifiestan hablar en castellano con los compañeros de trabajo y con quien les da trabajo pese a que sean catalanoparlantes. El uso del catalán se reduce a las actividades que realizan como trabajadores autónomos (venta ambulante, servicio doméstico), por cuenta propia y en relación con el público catalán (Solé, 1999).

\section{La inmigración femenina}

El concepto de raza no está, hoy en día, tan unido a las cuestiones relacionadas con la subordinación política, pero continúa siéndolo en términos de racismo, como yo he mencionado en otras publicaciones (Solé, 1995). Dado que la categoría de inmigración va substituyendo progresivamente a la de raza, a causa de los flujos migratorios y el peso que tiene la distancia cultural en relación con los procesos de integración, se puede hablar de la triple discriminación de las mujeres inmigrantes. Esta discriminación a tres dimensiones de las mujeres, se manifiesta en dos aspectos. En primer lugar, el carácter de su trabajo, una vez se instalan en la sociedad receptora: un trabajo en un mercado laboral segmentado por el género y segmentado por la etnicidad. En segundo lugar, la cuestión de la invisibilidad en la que se encuentran, tanto en la jerarquía laboral, de las tareas que realizan, como también en la vida social. Invisibilidad no significa solamente que la mayoría de mujeres inmigrantes quieran pasar desapercibidas para evitar el rechazo, sino que tampoco se las toma en consideración, ni en debates parlamentarios, ni, muchas veces, en los medios de comunicación. En fin, no forman parte, o parece que no formen parte, de la sociedad donde están trabajando y/o donde están residiendo.

Pueden distinguirse dos patrones de inmigración femenina. El primer patrón es el de la reunificación familiar: las mujeres que emigran hacia los países denominados «avanzados» porque el marido, o el hombre, o el padre, que es quien inicia la cadena migratoria para la familia, se ha instalado en un determinado país, tradicionalmente importador de mano de obra. El segundo patrón 
es el de inmigración de mujeres que inician la cadena migratoria hacia los países occidentales, que se han transformado de emisores de emigración a receptores. Son países que han recibido inmigración del sur de Europa, o que han sido imperios y reciben hoy población de sus ex colonias, población que se acoge a la ciudadanía en la metrópoli (Inglaterra, Portugal). Muchas mujeres inmigran solas a estos países en busca de mayores oportunidades laborales, mayor libertad y estima social.

Hay, pues, dos vertientes del problema: la subordinación en el mercado de trabajo y la marginación social. Aquí hay que mencionar la construcción social de la mujer inmigrante, por considerarla responsable de su situación marginal en el mundo laboral y social. Esta construcción social responde a los estereotipos y a los prejuicios que dominan en la sociedad receptora. De nuevo aparece un factor etnocéntrico en el planteamiento y en el tratamiento del tema y de los problemas con que debe enfrentarse la mujer inmigrante una vez se establece en una sociedad receptora.

Ahora bien, aparte de inducir a un replanteamiento teórico del tema, esta situación está avalada por hechos y por datos. Se observa como la mujer inmigrante ha ido adquiriendo, en los últimos veinte años, un protagonismo claro en las trayectorias migratorias. Muchas veces son ellas las que inician la trayectoria migratoria; no tan sólo el proyecto de emigrar de sus sociedades e inmigrar hacia otra sociedad, sino que también, en algunos casos, de arrastrar a marido e hijos e iniciar la cadena migratoria. Es decir, se invierten los papeles tradicionales de migración básicamente masculina, donde el hombre es el que protagoniza todo el proceso, tanto de migración como de instalación, de inserción y de integración en la sociedad receptora.

En muchos casos cabe señalar (nosotros lo hemos constatado para las mujeres filipinas y puede hacerse extensivo a las dominicanas y centroamericanas en general) que las mujeres no pueden ni tienen posibilidad de reclamar al marido y a los hijos, a su familia, debido al puesto de trabajo que alcanzan a ocupar, básicamente el servicio doméstico, que les proporciona escasos recursos. Los factores culturales del lugar de origen tienen también un papel importante, ya que impregnan el sistema de valores de su familia. Todo ello contribuye a que existan más dificultades que en el caso inverso: en el caso del hombre que reclama a mujer e hijos a que se reúnan con él.

Por otro lado, la invisibilidad característica de la inmigración, específicamente de la mujer inmigrante, desaparece cuando estallan brotes de racismo y surgen estallidos de actitudes xenófobas en las sociedades receptoras. La invisibilidad deja paso entonces a la presencia en los medios de comunicación de los inmigrantes y, en consecuencia, de la mujer inmigrante. Ahora bien, ésta continúa siendo, en relación o en comparación con los hombres, invisible en muchos casos por el tipo de trabajo que realizan: servicios de limpieza en empresas, atención a enfermos, atención a ancianos, cuidado de niños, servicio doméstico. El puesto de trabajo comporta muchas situaciones de clara discriminación, en términos salariales, pero especialmente en términos de condiciones laborales, ausencia de contrato, horas extras no pagadas, fines de semana 
disponibles, es decir, disponibilidad horaria que los empresarios no se atreverían nunca a pedir a un autóctono. Todas estas situaciones permanecen invisibles (a los ojos de los inspectores de trabajo, por ejemplo), porque, de acuerdo con el régimen laboral establecido, si no hay una denuncia explícita, que además sea corroborada por el propio empleador, es muy difícil detectar una situación de este tipo. En este sentido, continúa la situación de invisibilidad reforzada por la situación de discriminación laboral (Solé, 1999b).

Otra cuestión a tener en cuenta es la fuerte imbricación entre la subordinación en que se encuentran en el mercado de trabajo y la marginación, tanto social como de ellas mismas como personas. Esta imbricación, evidente para los inmigrantes, es diáfana para las mujeres, por el tipo de trabajo que generalmente realizan. Así, de la marginalidad de los puestos de trabajo que tienen como inmigrantes se deriva la marginalidad de ellas mismas como personas. No se las considera como trabajadoras imprescindibles para el proceso productivo, sino substitutivas o subsidiarias del trabajo masculino o del trabajo de mujeres autóctonas. De esta forma, se perpetúa la situación de discriminación que supone para muchas inmigrantes trabajar en actividades no cualificadas o, como nosotros hemos podido constatar, de cualificación inferior al nivel de estudios o de formación profesional que tienen. Esta situación es muy clara en el caso de las mujeres filipinas que nosotros pudimos entrevistar: están ocupadas en el servicio doméstico, o cuidan de enfermos, niños, etc., y, en cambio, tienen un nivel de estudios medio, son comadronas o son enfermeras, o son maestras, o a veces tienen estudios superiores, especialmente las mujeres de África occidental (Solé, 1994).

Así pues, se forma un círculo vicioso entre subordinación laboral y marginación social que resulta reforzado por una serie de estereotipos, de prejuicios, sobre las mujeres inmigrantes. Se las considera afortunadas por el hecho de poder tener un salario algo más elevado que en su lugar de origen. No se tiene en cuenta, sin embargo, el coste personal que supone para muchas de ellas dejar la familia en el lugar de origen, dejar su entorno cultural, habida cuenta de que las mujeres que emigran de sus países hacia las sociedades occidentales, entre ellas la nuestra, no son mujeres que se hallen en una situación de precariedad económica extrema. Muchas tienen recursos, como es un nivel de formación relativamente alto, que hace que sus expectativas de una vida mejor o de un nivel o calidad de vida más elevado, no solamente en términos monetarios, sino también de un mayor grado de libertad, las lleven a iniciar la trayectoria migratoria. Ciertamente, muchas veces esta iniciativa responde a una estrategia familiar: no es ella quien individualmente decide irse de su país. Pero un conjunto de factores históricos, económicos del lugar de origen condicionan sus posibilidades y capacidades. Además, la demanda en los países receptores de inmigración (especialmente los occidentales) tiende hacia un aumento de demanda en el sector de los servicios, especialmente el sector de los servicios personales. Por lo tanto, el estereotipo de la visión de la mujer como víctima, o de la mujer inmigrante como afortunada, por escapar de una situación de pobreza o miseria, debería ser replanteada, cuando no rechazada. 


\section{Bibliografía}

ARAGÓN, R.J.; ChOZAS, J. (1993). La regularización de los inmigrantes, 1991-1992. Madrid: Ministerio de Trabajo y Seguridad Social.

BoIX, E. (1993). Triar no és trair. Barcelona: Edicions 62.

IzQUIERDO, A. (1992). La inmigración en España 1980-1990. Madrid: Ministerio de Trabajo y Seguridad Social. Informes núm. 17.

SABEL, Ch. (1985). Trabajo y Política. Madrid: Ministerio de Trabajo.

SOLÉ, C. (1981). La integración sociocultural de los inmigrantes en Cataluña. Madrid: CIS.

- (1988). Catalunya: Societat receptora d'immigrants. Barcelona: Institut d'Estudis Catalans.

- (1994). La mujer inmigrante. Madrid: Instituto de la Mujer.

- (1995). Discriminación racial en el mercado de trabajo. Madrid: CES.

- (1999a). «L'ús instrumental del català a Ciutat Vella (Barcelona)». Tallers de sociolingüistica, núm. 14. Barcelona.

- (1999b). "La inmigració femenina en l'era de la globalització». En RoQue, Maria Àngels (dir.). Dona i migració de la Mediterrània occidental. Barcelona: Proa.

SolÉ, C. (y colaboradores) (1992). Estudi sobre la realitat social de les minories ètniques al Districte de Ciutat Vella. Barcelona: Ajuntament de Barcelona.

Subirats, M. (1992). Enquesta Metropolitana de Barcelona, vol. 4. Educació i hàbits culturals. Barcelona: Institut d'Estudis Metropolitans. 